\title{
PERSPECTIVES
}

\section{Natural inspirations for metal-ligand cooperative catalysis}

\section{Matthew D. Wodrich and Xile Hu}

Abstract | In conventional homogeneous catalysis, supporting ligands act as spectators that do not interact directly with substrates. However, in metal-ligand cooperative catalysis, ligands are involved in facilitating reaction pathways that would be less favourable were they to occur solely at the metal centre. This catalysis paradigm has been known for some time, in part because it is at play in enzyme catalysis. For example, studies of hydrogenative and dehydrogenative enzymes have revealed striking details of metal-ligand cooperative catalysis that involve functional groups proximal to metal active sites. In addition to the more well-known [FeFe]-hydrogenase and [NiFe]-hydrogenase enzymes, [Fe]-hydrogenase, lactate racemase and alcohol dehydrogenase each makes use of cooperative catalysis. This Perspective highlights these enzymatic examples of metal-ligand cooperative catalysis and describes functional bioinspired molecular catalysts that also make use of these motifs. Although progress has been made in developing molecular catalysts, considerable challenges will need to be addressed before we have synthetic catalysts of practical value.

The field of catalysis, broadly defined, attracts considerable attention in both academia and industry. To most chemists, homogeneous catalysis has historically been considered to involve one or two metal atoms to which ligands are attached. These ligands shape the catalytic environment both physically, through steric and other noncovalent interactions, and electronically, through inductive and resonance effects on the metal centre (BOX 1). Traditionally, the ligands are not directly involved in the reaction that is being catalysed, which occurs only at the metal centre. Enzymatic catalysis typically takes advantage of more sophisticated processes for facilitating the chemical reactions that are essential for life. Once again, they tend to feature one or more metal atoms surrounded by a sphere of ligands, which may take the form of amino acids, organic cofactors and other typical inorganic ligands. However, many enzymes have evolved to make use of both the metal centre and the surrounding ligand environment in performing catalysis (BOX 1). Referred to as 'cooperative catalysis' (REFS 1,2), this paradigm can also be incorporated into designs of new synthetic catalysts, wherein both the metal centre(s) and the surrounding abiotic ligands act to mediate an otherwise slow reaction. Among other aspects, some perceived kinetic advantages of cooperative catalysis include the creation of an additional substrate binding site, the possibility of having Lewis and/or Brønsted acidic and basic sites within the same catalyst and the preorganization of the transition state.

Metal-ligand cooperation has long been recognized as being central to the function of metalloproteins, with one prominent example being the binding of $\mathrm{O}_{2}$ in myoglobin, wherein a distal histidine residue forms a hydrogen bond to $\mathrm{Fe}$-coordinated $\mathrm{O}_{2}$. This motif has been replicated in synthetic systems that feature so-called picket-fence and picnic-basket porphyrinato complexes decorated with hydrogen-bond donors ${ }^{3,4}$. However, beyond hydrogen bonding, direct ligand participation in bond-breaking and bond-forming steps in enzyme catalysis has only begun to be elucidated. Recent studies have revealed or confirmed that such ligand participation helps allow for hydrogen metabolism and dehydrogenation reactions in a number of enzymes such as [FeFe]hydrogenase, [FeNi]-hydrogenase,

[Fe]-hydrogenase, lactate racemase and alcohol dehydrogenase. In this Perspective, we discuss these examples of biological cooperative catalysis and how, inspired by these naturally occurring systems, chemists have sought to create functional analogues. We also speculate that cooperative catalysis may be at play in $[\mathrm{NiFe}]$-carbon monoxide dehydrogenase ([NiFe]-CODHase), which may inspire the design of synthetic $\mathrm{CO}_{2}$ reduction catalysts making use of these motifs. Each section in the following discussion is devoted to an enzyme and its synthetic models.

\section{[FeFe]- and [NiFe]-hydrogenase}

Hydrogenases are enzymes that catalyse the production and utilization of $\mathrm{H}_{2}$ (REF. 5). Three variants of hydrogenase enzymes exist which are named [FeFe]-, [NiFe]- and [Fe]-hydrogenase because their active sites feature these catalytic metals ${ }^{6}$. The metal centres in both [FeFe]- and [NiFe]-hydrogenase are coordinated to cysteine residues and small inorganic $\left(\mathrm{CO}\right.$ and $\left.\mathrm{CN}^{-}\right)$ligands that interact with the surrounding protein environment through hydrogen-bonding interactions (FIG. 1a,b). Each of the three different active sites features one metal coordination site that is accessible for the binding of either $\mathrm{H}_{2}$ or $\mathrm{H}^{+}$ substrates, with $\mathrm{H}^{+}$substrates being bound in the form of a hydride ${ }^{7}$. Certain structural moieties, such as the bis(sulfidomethyl) amine (azadithiolate, $\left.\mathrm{HN}\left(\mathrm{CH}_{2} \mathrm{~S}^{-}\right)_{2}\right)$ ligand in $[\mathrm{FeFe}]$-hydrogenase ${ }^{8,9}$ and the terminal cysteine residues in $[\mathrm{NiFe}]$-hydrogenase ${ }^{10}$, have long been implicated as internal bases - groups that relay $\mathrm{H}^{+}$and facilitate $\mathrm{H}_{2}$ activation or production ${ }^{6}$. These two enzymes, which interconvert $\mathrm{H}_{2}$, protons and electrons, are now described in terms of these second coordination sphere effects.

Elegant studies involving the insertion of synthetic mimics of the [FeFe]-hydrogenase active site into the cofactor-free apo-form of [FeFe]-hydrogenase have afforded semisynthetic enzymes with varying activities $^{11-15}$. Full activity of the native enzyme was achieved only by the use of a mimic that features an azadithiolate ligand bridging two Fe centres. This result indirectly 


\section{Box 1 | Classical and cooperative catalysis}

In classical transition metal catalysis (see the figure, part a), only the metal is involved in the reaction, with the ligand(s) serving mainly to engender certain electronic and steric properties on the metal centre. In cooperative catalysis (see the figure, part b), both the metal centre and one or more ligands are directly involved in the reactions. Interactions with the substrate may involve hydrogen bonding, dative bonding and/or electrostatic interactions.

a

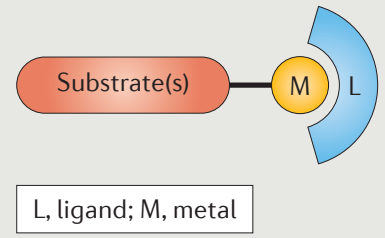

b

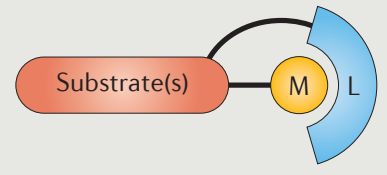

confirms the presence of this ligand in the active site of the [FeFe]-hydrogenases, providing strong evidence that the $\mathrm{H}_{2}$ formation and oxidation mechanisms involve the azadithiolate amine in the second coordination sphere relaying $\mathrm{H}^{+}$to and from the active site (FIG. 1a). A similar study on $[\mathrm{NiFe}]$-hydrogenase ${ }^{10}$ used site-directed mutagenesis to show that a $\mathrm{N}$ atom in a strictly conserved arginine residue acts as a general base for $\mathrm{H}_{2}$ activation. Nature judiciously places this atom $4.5 \AA$ above the $\mathrm{Fe}$ and Ni centres in the enzyme, the bimetallic site that eventually receives the substrate, in this case in the form of $\mathrm{H}^{-}$(FIG. 1 b). Although the role of azadithiolate as a proximal base in $[\mathrm{FeFe}]$-hydrogenases has been firmly established $^{6,9}$, the analogous role of Arg509 in the [NiFe]-hydrogenase catalytic mechanism is only a recent proposal that awaits further confirmation.

\section{[Fe]-hydrogenase}

[Fe]-hydrogenase is different from the other hydrogenase enzymes in that its active site contains only a single metal centre, which takes the form of a cysteine-ligated Fe-guanylylpyridinol (FeGP) cofactor ${ }^{16,17}$ (FIG. 2a). The enzyme does not perform redox but rather catalyses the reversible transfer of $\mathrm{H}^{-}$from $\mathrm{H}_{2}$ to a formamidinium group in methenyltetrahydromethanopterin ${ }^{6}$ (methenyl- $\mathrm{H}_{4} \mathrm{MPT}^{+}$, FIG. $2 \mathrm{~b}$ ), a process that is essential for metabolism in hydrogenotrophic methanogenic archaea ${ }^{17,18}$. The [Fe]-hydrogenase active site consists of an $\mathrm{Fe}^{\mathrm{II}}$ centre coordinated to the sulfur atom of a cysteine residue, two cis-CO ligands and the acyl $\mathrm{C}$ atom and pyridyl $\mathrm{N}$ atom of the pyridinol ligand. An open sixth coordination site is presumably the site of $\mathrm{H}_{2}$ binding. $\mathrm{X}$-ray crystal structure analysis ${ }^{19}$ and computational studies ${ }^{20-22}$ suggest that $\mathrm{H}_{2}$ heterolysis occurs between the pyridonate $\mathrm{O}$ atom, the Fe centre and the carbocation-like formamidinium in methenyl- $\mathrm{H}_{4} \mathrm{MPT}^{+}$.
Thus, akin to the [NiFe]- and [ $\mathrm{FeFe}]-$ hydrogenases, $[\mathrm{Fe}]$-hydrogenase also participates in cooperative catalysis by means of functional groups in its second coordination sphere that relay $\mathrm{H}^{+}$.

The $[\mathrm{Fe}]$-hydrogenase holoenzyme can be reconstituted from its apo-enzyme and the isolated cofactor. This gave synthetic chemists hope that artificial cofactors might also be amenable to the reconstitution procedure and afford new species with potentially high activities. Such semisynthetic enzymes provide an ideal tool for studying the mechanistic role played by ancillary moieties located in the second coordination sphere. One such structurefunction relationship was uncovered by preparing semisynthetic [Fe]-hydrogenases by use of the synthetic model complexes 1 and 2 (REF. 23) (FIG. 2C). Although neither of these synthetic cofactors showed activity in the absence of a protein environment, the semisynthetic enzyme derived from 1 was found to have turnover frequencies of $2 \mathrm{~s}^{-1}$ for the reduction of methenyl- $\mathrm{H}_{4} \mathrm{MPT}^{+}$ with $\mathrm{H}_{2}$. When the 2-hydroxyl group in 1 is replaced with a 2-methoxy group, as it is in 2, the model is still incorporated into the protein, but the product is essentially inactive. Thus, the pyridone/pyridinol $\mathrm{O} / \mathrm{OH}$ group located within the second coordination sphere is critical to the function of these semisynthetic enzymes. This result can logically be extended to the native $[\mathrm{Fe}]$ hydrogenase, implicating an important mechanistic role for the $\mathrm{O} / \mathrm{OH}$ group of the FeGP cofactor. Density functional theory computations on intermediates in the active semisynthetic enzyme catalytic cycle afford a free energy profile indicating that deprotonation of the 2-hydroxyl group lowers the barrier to $\mathrm{H}_{2}$ heterolysis by $\sim 80 \mathrm{~kJ} \mathrm{~mol}^{-1}$ (from 137 to $59 \mathrm{~kJ} \mathrm{~mol}^{-1}$ ). This lower-energy pathway is made possible by cooperative catalysis (FIG. 2d), something that the semisynthetic enzyme that contains the 2-methoxy-containing cofactor cannot perform. As a result, the enzyme reconstituted using 2 is unable to access a low-energy pathway to $\mathrm{H}_{2}$ heterolysis, such that it has no detectable activity. The reconstitution study provides further evidence that $[\mathrm{Fe}]$-hydrogenase operates through cooperative catalysis in which a second coordination sphere heteroatomcontaining group acts as an internal base.

Many early synthetic models for [Fe]hydrogenase, some closely resembling the active site of the native enzyme, were unable to catalyse either $\mathrm{H}_{2}$ heterolysis or activation ${ }^{24}$. Guided by mechanistic insights from the reconstitution studies, we used density functional theory computations to predict the activity of several synthetically viable biomimetic complexes, finding that species bearing a pendant amine were most likely to activate $\mathrm{H}_{2}$ (REF. 25). A close analogue of the most promising computational model was synthesized, with this catalyst candidate featuring biomimetic pyridinylacyl and CO ligands in addition to a diphosphine ligand with a noncoordinated amine $\mathrm{a}^{26}$ (3, FIG. 2e). The amine-diphosphine, although not structurally relevant to [Fe]-hydrogenase, is bioinspired in that it is reminiscent of the azadithiolate cofactor in [FeFe]-hydrogenase. Thus, the amine-diphosphine serves as a $\mathrm{H}^{+}$ acceptor in the heterolytic activation of $\mathrm{H}_{2}$ by cooperative catalysis. The presence of an exotic and abiological amine-diphosphine ligand in 3 means that it is not considered a structural mimic of [Fe]-hydrogenase, although it is able to cleave $\mathrm{H}_{2}$ and deliver it to an aldehyde. This classification is not necessarily a bad thing, because an exciting goal of biomimetic chemistry is to develop synthetically useful catalysts, regardless of whether the ligands are biologically relevant. The main limitation of 3 is the low stability of the ammonium hydride intermediates generated after $\mathrm{H}_{2}$ activation, such that the efficiency of $\mathbf{3}$ for hydrogenation is modest. Further work in this area would target more robust derivatives that are nevertheless able to transfer $\mathrm{H}_{2}$ (in the form of $\mathrm{H}^{+}$and $\mathrm{H}^{-}$) to polar unsaturated substrates.

The structural and mechanistic features of [Fe]-hydrogenase have inspired the design of Fe-based catalysts and have motivated many chemists to incorporate the 2-hydroxypyridine motif into cooperative catalysts featuring other metals and co-ligands ${ }^{27}$. Thus far, such work has been limited to complexes of $\mathrm{Ru}$ or Ir. In the spirit of bioinspired catalysis, extension to earth-abundant metal species is warranted. 


\section{Lactate racemase}

Lactate racemase catalyses the interconversion of the $\mathrm{D}$ - and L-lactic acid enantiomers at an active site featuring a unique Ni pincer ${ }^{28-30}$ (FIG. 3Aa). This intriguing cofactor consists of a Ni centre coordinated to a niacin-derived SCS-donor ligand. The whole complex is anchored to the protein backbone through a lysine residue in the pincer and a histidine residue bonded to Ni (REFS. 28-30). The nature of the catalytic intermediates observed led early studies to conclude that the mechanism may involve an internal $\mathrm{H}^{-}$transfer ${ }^{31}$, a hypothesis that could not be developed owing to the lack of structural data necessary for the proposal of a full mechanism. However, the recently solved X-ray crystal structure of lactate racemase ${ }^{28}$ afforded the necessary atomic coordinates from which density functional theory studies could shed light on specific details, including the deprotonation of $\mathrm{D} / \mathrm{L}$-lactate by a proximal histidine residue and $\mathrm{H}^{-}$transfer to the Ni-bound $\mathrm{C}$ atom ${ }^{32,33}$ (FIG. 3Ab). The acetyl group of the metastable pyruvate (dehydrogenated lactate) intermediate can undergo rotation, after which it can accept $\mathrm{H}^{+}$and $\mathrm{H}^{-}$to form the other enantiomer of lactate. Overall, the lactate racemase mechanism is a further example of direct ligand participation in enzyme catalysis. In the proposed mechanism, the metal centre (Ni) does not bind to the substrate; instead, it serves to fix the ligand in the active site and tune the properties of the ligands. In this sense, the enzyme is not strictly a cooperative catalyst in which both metal and ligands are directly involved in the reactions. However, the binding and activation of the substrate by the Ni centre in a transition state or intermediate cannot be ruled out. Although the participation of lactate racemase in cooperative catalysis is currently only hypothetical, its ligand involvement is reminiscent of those in cooperative enzymes.

\section{Alcohol dehydrogenase}

Alcohol dehydrogenase is a $\mathrm{Zn}$-dependent enzyme that reversibly oxidizes alcohols to aldehydes or ketones by means of cooperative $\mathrm{H}^{+}$and $\mathrm{H}^{-}$transfers. As is the case with lactate racemase, it is not the metal site but rather an organic fragment - here, nicotinamide adenine dinucleotide $\left(\mathrm{NAD}^{+}\right)$- that accepts $\mathrm{H}^{-}$from the alcohol $\mathrm{C}$ atom (FIG. 3Ba). Before this can happen, the $\mathrm{Zn}^{2+}$ centre must coordinate to the hydroxyl group, polarizing it such that it can donate $\mathrm{H}^{+}$to a proximal serine residue $\mathrm{e}^{34-36}$. The serine residue is part of a hydrogen-bonding network that also features the ribose group and a neighbouring histidine $e^{37,38}$ (FIG. 3Bb) that ultimately shuttles $\mathrm{H}^{+}$to the protein surface. Overall, it is the combined action of $\mathrm{Zn}^{2+}$, along with the $\mathrm{H}^{+}$and $\mathrm{H}^{-}$acceptors, that serves to dehydrogenate, for example, benzyl alcohols to give the corresponding benzaldehydes. Although several isozymes exist, the crystal structure of liver alcohol dehydrogenase has been determined, revealing that the active site features $\mathrm{Zn}^{2+}$ bound to two cysteine residues and one histidine residue, with the tetrahedral coordination sphere also including either a substrate or $\mathrm{H}_{2} \mathrm{O}$ molecule.

The unique $\mathrm{Ni}$ (SCS) active site of lactate racemase inspired immediate interest in developing synthetic models of this motif. Our group has prepared 4 and 5 (REF. 39) (FIG. 3C), two Ni complexes of SCS pincer ligands very similar to the enzyme active site. The difference between the two biomimics 4 and 5 lies in the pincer backbone, with one possessing a pyridine fragment and the other an $\mathrm{N}$-methylpyridinium. Probing the changes in reactivity that result from this subtle difference might reveal important information surrounding the nature of the cooperative catalysis that occurs within the enzyme. Indeed, the $\mathrm{N}$-methylpyridinium derivative 5 readily effects dehydrogenation of benzyl alcohol, whereas pyridine complex 4 exhibits negligible activity. Density functional theory computations revealed that a mechanism involving formation of a $\mathrm{Ni}$ alkoxo followed by $\beta-\mathrm{H}$ elimination is energetically inaccessible for both model complexes. A more energetically viable pathway is one in which the $\mathrm{H}^{-}$attacks the $\mathrm{N}$-heterocycle at one of its $\mathrm{C}$ atoms, with attack at the 2 and 4 positions being most likely. Attack at the 4 position (FIG. 3D), despite being slightly less favourable, is most relevant to the enzyme mechanism. In either case, $\mathrm{H}^{-}$addition to the electron-poor pyridinium ring in 5 is accompanied by a transition-state barrier $\sim 10 \mathrm{kcal} \mathrm{mol}^{-1}$ smaller than that for the corresponding addition to the pyridine in 4. In both cases, this biomimic of lactate racemase successfully modelled the ligand participation feature of the enzyme.

Unlike the enzyme, complex 5 mediates dehydrogenation only irreversibly, precluding it from being an effective racemization catalyst. Substantial differences exist between the Ni coordination environment at the enzyme active site and the structure of 5 . These differences, together with second coordination sphere effects exerted by residues in the vicinity of the active site, may be responsible for the observed reactivity difference. Although a synthetically useful high-fidelity mimic of lactate racemase remains elusive, there is a 'ferraquinone' catalyst that can both dehydrogenate alcohols and activate $\mathrm{H}_{2}$ (6, FIG. 3E). Both reactions are a result of cooperativity between the Fe centre and the $\mathrm{C}$ atom bonded to it, and the
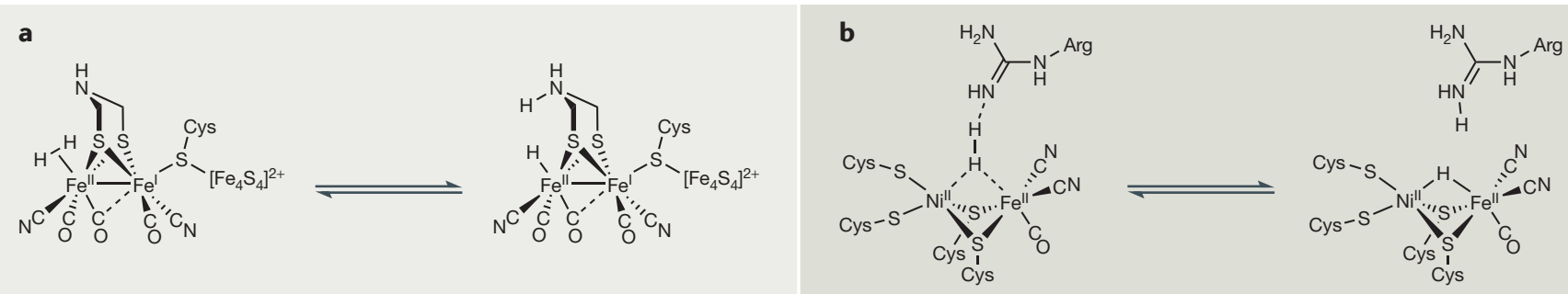

Figure 1 | Cooperative catalysis in [FeFe]- and [NiFe]-hydrogenases enables the heterolysis of $\mathbf{H}_{2}$. a | The [FeFe]-hydrogenase active site contains a secondary amine that deprotonates $\mathrm{H}_{2}$ coordinated to Fe" (REF. 9). b | Similarly, $\mathrm{H}_{2}$ coordinated at the [NiFe]-hydrogenase active site is cleaved by the guanidine moiety in the proximal Arg509 residue ${ }^{9,10}$. The utility of pendant amines as $\mathrm{H}^{+}$relays in homogeneous catalysts has long been recognized. This theme continues to be rediscovered as new synthetic enzyme models appear that incorporate these, or chemically similar, components into their second coordination spheres ${ }^{8,52-54}$. Many biomimetic catalysts inspired by the hydrogenases are designed to perform cooperative catalysis in much the same way as enzymes. The biomimetic chemistry of hydrogenases is now well established, and readers wishing to learn more about these themes are directed to several useful reviews $s^{6,8,9,52,55-58}$. 

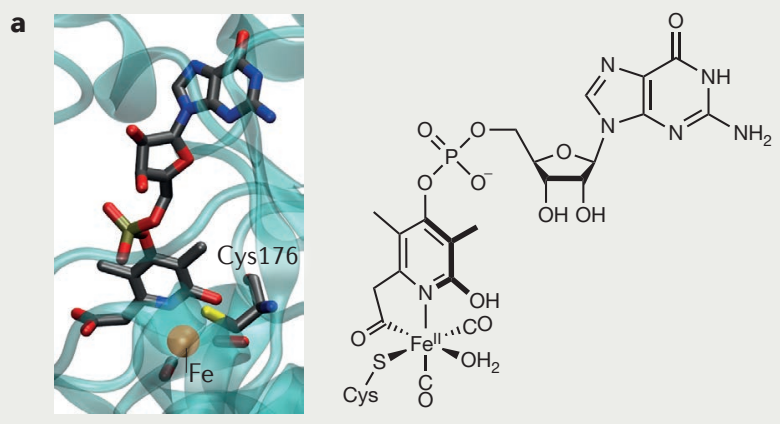

b

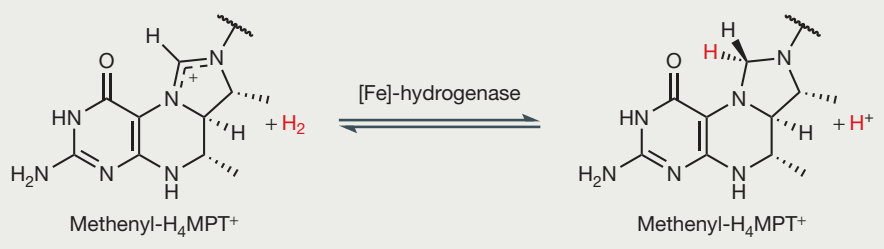

C
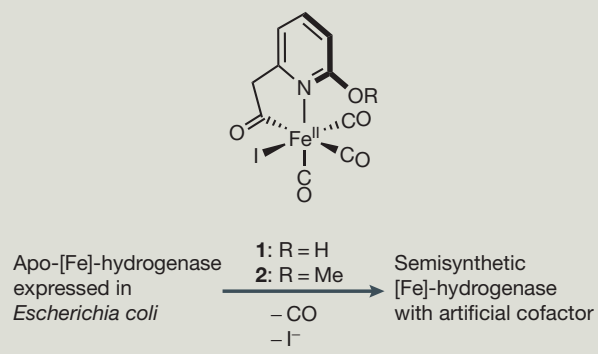

d

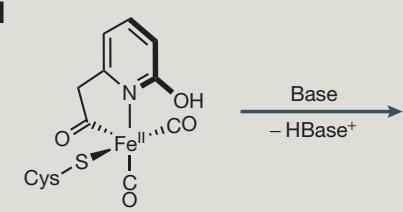

$\mathbf{e}$

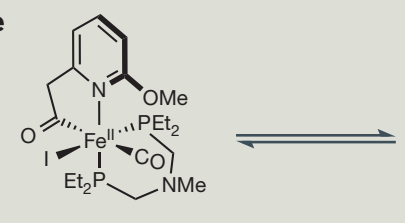

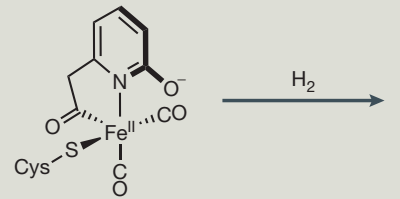



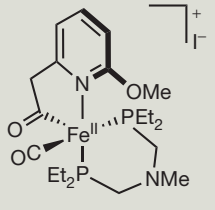

$3^{\prime}$

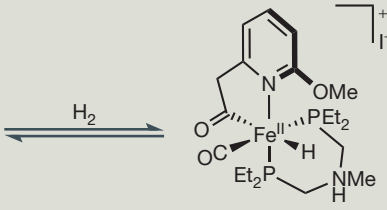

$3 \mathrm{H}_{2}$

Figure $2 \mid$ [Fe]-hydrogenase and its model compounds. a | The active site of [Fe]-hydrogenase is tethered to the protein backbone through a single cysteine residue. The Fe site is octahedral, but the aquo ligand can leave in order to vacate a binding site for $\mathrm{H}_{2}\left(\right.$ Protein Data Bank identifier: 3 DAG ${ }^{18}$. b | The enzyme catalyses the stereospecific delivery of $\mathrm{H}^{-}$to the pro- $R$ face of methenyltetrahydromethanopterin $\left(\text { methenyl- }_{4} \mathrm{MPT}^{+}\right)^{23}$. $\mathbf{c} \mid \mathrm{The} \mathrm{cofactor}-\mathrm{free}$ apo-form of [Fe]-hydrogenase (apo-[Fe]-hydrogenase) can be used to reconstitute, by means of synthetic ferrous carbonyls 1 or 2, semisynthetic enzymes in which the active sites feature the synthetic fragments ${ }^{23}$. $\mathbf{d} \mid$ The semisynthetic enzyme derived from 1 performs $\mathrm{H}_{2}$ heterolysis by the use of metal-ligand cooperativity ${ }^{23}$. The protein environment is omitted for clarity. e $\mid$ A synthetic model of the [Fe]-hydrogenase active site (3) heterolyses $\mathrm{H}_{2}$ between its basic tertiary amine site and Lewis acidic Fe" site ${ }^{26}$. In the protein structure, C (grey), N (blue), O (red), P (gold), S (yellow) and Fe (orange) atoms are shown in their respective colours, whereas $\mathrm{H}$ atoms are removed for clarity. Part a was drawn using data in REF. 18.

'ferrahydroquinone' $6 \mathrm{H}_{2}$ is generated in either case $\mathrm{e}^{40}$. Although the ligands present in 6 are quite different from those bound to $\mathrm{Ni}$ in lactate racemase, the direct involvement of the pincer ligand bears resemblance to the native mechanism.

Alcohol dehydrogenase has also served as an inspiration for transition-metal-based catalysts for transfer hydrogenation reactions. For example, bioinspired catalysts 7 and 8 feature a benzimidazolium moiety (a strong $\mathrm{H}^{-}$acceptor) tethered to a 1,10-phenanthroline chelated to $\mathrm{RhCp}^{*}$ or IrCp $\mathrm{p}^{\star}$ fragments ${ }^{41}$ (FIG. 3F). The catalysts are active for imine hydrogenation and are thought to operate by a mechanism similar to that of alcohol dehydrogenase. Complexes 7 and 8 can hardly be considered biomimetics, as they contain either a Rh or an Ir metal centre (as opposed to $\mathrm{Zn}$ ) along with $\mathrm{Cp}^{*-}$ and 1,10-phenanthroline ligands instead of donors more relevant to amino acids. Thus, an interesting challenge would be to develop $\mathrm{Zn}$ variants of such complexes with comparable reactivities.

\section{[NiFe]-CODHase}

There is considerable interest in better understanding the chemistry of [NiFe]-CODHase, an enzyme that catalyses the $2 \mathrm{H}^{+} / 2 \mathrm{e}^{-}$reduction of $\mathrm{CO}_{2}$ to give $\mathrm{CO}$ and $\mathrm{H}_{2} \mathrm{O}$ (REF. 42). Such studies are motivated by potential applications of relevance to energy and the environment. The [NiFe]-CODHase active site contains a unique $\left[\mathrm{NiFe}_{4} \mathrm{~S}_{5}\right]$ cluster that can exist in the multiple oxidation states (each differing by one electron) required to perform $\mathrm{CO}_{2}$ reduction $^{43-47}$ (FIG. 4a). The atomicresolution X-ray crystal structure of $\mathrm{CO}_{2}$-bound [NiFe]-CODHase reveals a bent $\mathrm{CO}_{2}{ }^{2-}$ ligand, the $\mathrm{C}$ atom of which coordinates to $\mathrm{Ni}$, while an $\mathrm{O}$ atom binds $\mathrm{Fe}$ (REF. 47). The charge on the ligand is inferred from the $\mathrm{O}-\mathrm{C}-\mathrm{O}$ angle $\left(\sim 117^{\circ}\right)$, which is similar to that in $\mathrm{HCO}_{2}^{-}$and is reflective of $s p^{2}$ hybridization at the $\mathrm{C}$ atom (FIG. 4b). The $\mathrm{CO}_{2}{ }^{2-}$ ligand interacts with the protein matrix through hydrogen bonds to His93 (2.72 $\AA$ ) and Lys563 (2.70 $)$. The key step in the conversion of bound $\mathrm{CO}_{2}{ }^{2-}$ to $\mathrm{CO}$ is the cleavage of the $\mathrm{C}-\mathrm{O}$ single bond in the $\mathrm{Ni}-\mathrm{C}-\mathrm{O}-\mathrm{Fe}$ moiety, a step proposed to occur after protonation of the $\mathrm{O}$ atom. Considering the importance of $\mathrm{H}^{+}$relays in hydrogenases, it seems reasonable to ask whether a second coordination sphere acid-base group can shuttle $\mathrm{H}^{+}$to and from the [NiFe]-CODHase active site. One plausible candidate is Lys563, a highly conserved residue that apparently plays an important role in catalysis ${ }^{48}$. Although still speculative, this proposed cooperative catalysis (FIG. 4b) provides food for thought in the design of synthetic [NiFe]-CODHase mimics. Experience in preparing synthetic models of $\mathrm{Fe}-\mathrm{S}$ proteins ${ }^{49}$ would suggest that faithful structural models of the complete clusters are very difficult to make. Nevertheless, progress has been made in mimicking part of the cluster ${ }^{42,49}$, although no [NiFe]-CODHase structural models contain an internal $\mathrm{H}^{+}$relay and none are competent electrocatalysts for the interconversion of $\mathrm{CO}$ and $\mathrm{CO}_{2}$ (REF. 42). However, two archetypal macrocycle complexes of Ni (9, FIG. 4c) and Fe (10) feature built-in hydrogen-bonding 
Aa

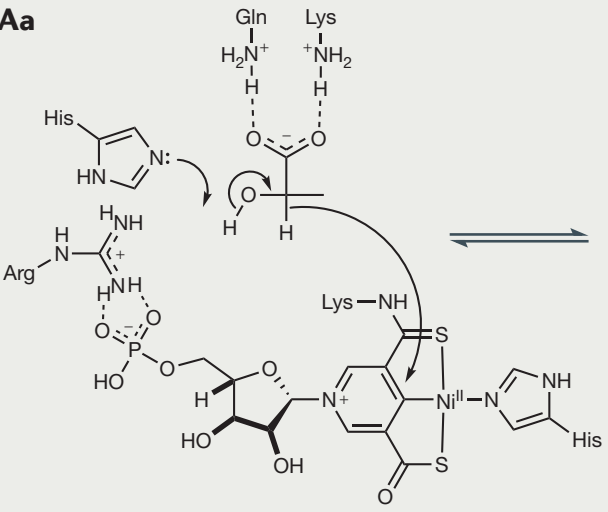

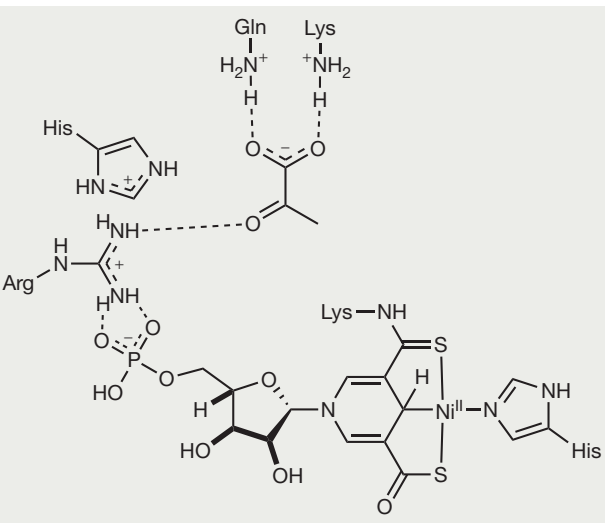

Ab

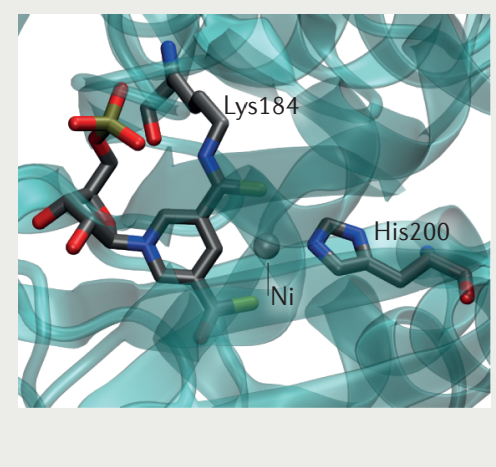

Ba

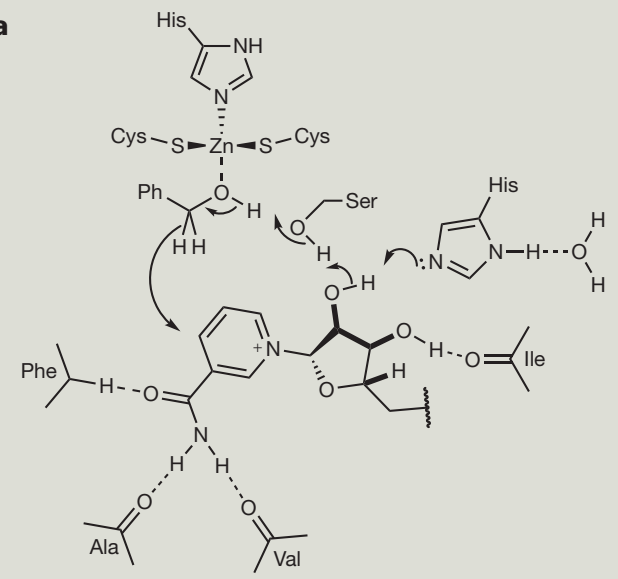

C<smiles>CCNC(=S)c1cncc2c(=S)sn(Cl)c12</smiles><smiles>CCNC(=S)c1cn(C)cc2c(C(=S)NCC)c3c(c1-2)[N+](Cl)(Cl)S3</smiles>

E

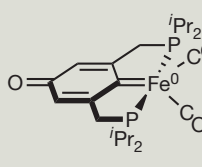

6
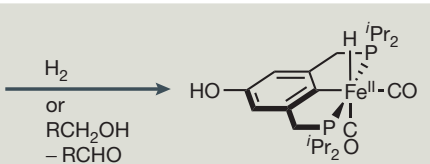

$6 \mathrm{H}_{2}$
Bb

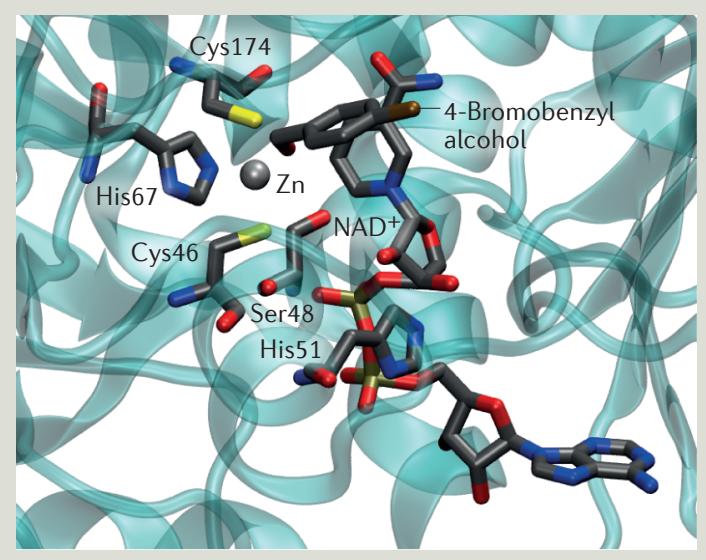

D

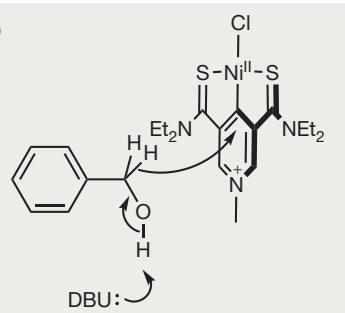
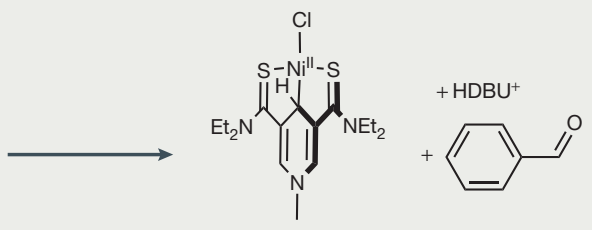

$\mathbf{F}$



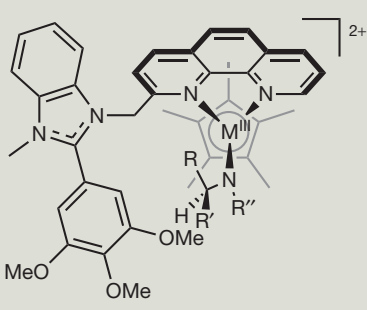

Figure 3 | Some features of the lactate racemase and alcohol dehydrogenase active sites have been replicated in synthetic mimics. Aa | The active site of lactate racemase features a single square planar organonickel cofactor bound to a histidine residue ${ }^{32,33}$. Ab $\mid$ The enzyme is proposed to reversibly dehydrogenate lactate by transferring $\mathrm{H}^{-}$to the $\mathrm{Ni}$-bound $\mathrm{C}$ atom, and $\mathrm{H}^{+}$to a histidine residue (Protein Data Bank identifier: $5 \mathrm{HUQ}$ ). Ba $\mid$ The active site of alcohol dehydrogenase features $\mathrm{Zn}^{2+}$ bound to one histidine residue and two cysteine residues, and here the substrate benzyl alcohol occupies the fourth coordination site ${ }^{36-38}$. $\mathrm{Bb} \mid 4$-Bromobenzyl alcohol, when bound to $\mathrm{Zn}^{2+}$, is susceptible to $\mathrm{H}^{-}$and $\mathrm{H}^{+}$abstraction by proximal $\mathrm{NAD}^{+}$and serine residues, respectively (PDB ID: 1 HLD). C | Synthetic models of the lactate racemase active site are pictured, these reproducing the catalytic Ni(SCS) motif. Models 4 and 5 feature pyridine and $\mathrm{N}$-methylpyridinium moieties, respectively ${ }^{39}$. D | Density functional theory computations for catalyst 5 predict that it performs alcohol dehydrogenation by means of metal-ligand cooperativity, with the pincer ligand accepting $\mathrm{H}^{-}$at either the 4 position (pictured) or the 2 position ${ }^{39}$. E The Fe ${ }^{0}$ ferraquinone 6 can activate $\mathrm{H}_{2}$ and dehydrogenate primary alcohols to aldehydes, thereby affording ferrahydroquinone $6 \mathrm{H}_{2}(\mathrm{REF}$. 40 ). F | Half-sandwich complexes of $\mathrm{Rh}(7)$ and $\operatorname{Ir}(8)$ featuring a tethered dihydroimidazole derivative catalysing $\mathrm{H}_{2}$ transfer to imines ${ }^{41}$. These bioinspired compounds feature organic $\mathrm{H}^{-}$and $\mathrm{H}^{+}$shuttles reminiscent of alcohol dehydrogenase. In the protein structures, $\mathrm{C}$ (grey), $\mathrm{N}$ (blue), $\mathrm{O}$ (red), $\mathrm{P}$ (gold) and S (yellow) atoms are shown in their respective colours, whereas $\mathrm{H}$ atoms are removed for clarity. The metal atoms are drawn as grey spheres and the protein scaffold is rendered in teal. DBU, 1,8-diazabicyclo[5.4.0]undec-7-ene. Part Ab was drawn using data in REF. 28. Part Bb was drawn using data in REF. 36. 
a

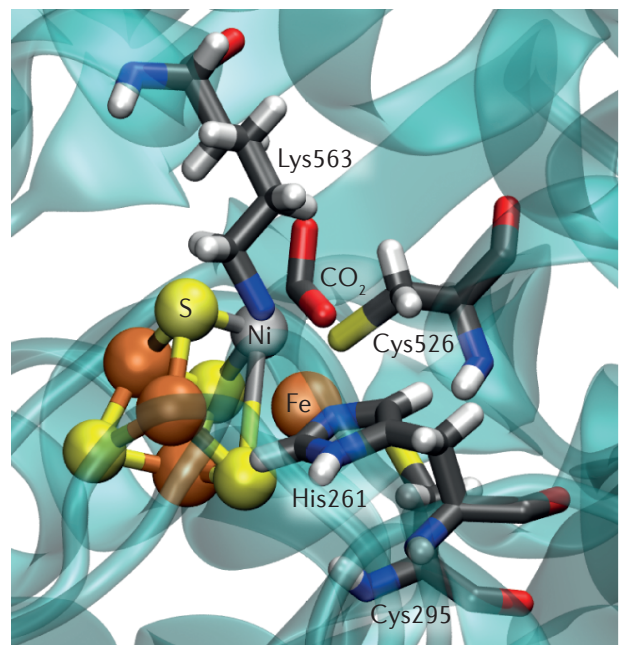

b

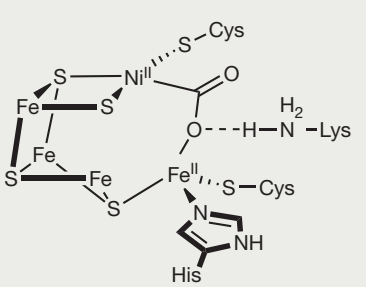

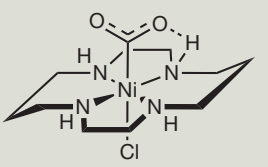
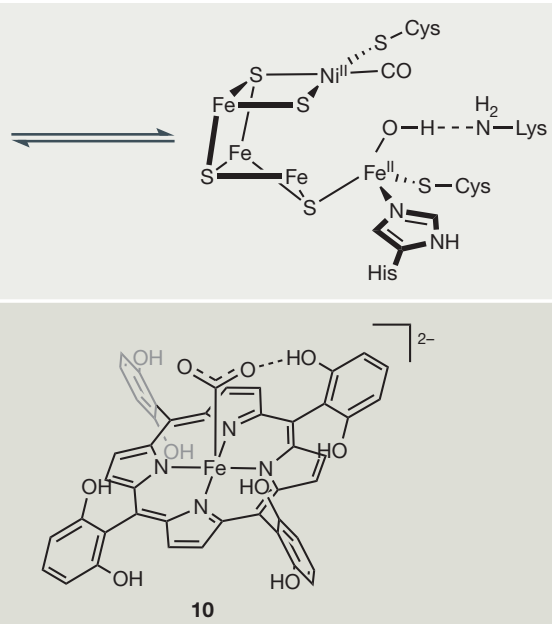

Figure 4 | [NiFe]-carbon monoxide dehydrogenase, [Ni(cyclam) Cl] ${ }^{+}$and an Fe porphyrinate each catalyse $\mathrm{CO}_{2}$ electroreduction to $\mathrm{CO}$. a | The enzyme active site features a distorted metallocubane, with the $\mathrm{Ni}$ apex binding the $\mathrm{C}$ atom and a further Fe site binding an $\mathrm{O}$ atom of the $\mathrm{CO}_{2}$ substrate, present here as a $\mathrm{CO}_{2}{ }_{2}^{2-}$ ligand (Protein Data Bank identifier: 4UDX). b | Protonation of the Fe-bound $\mathrm{O}$ atom is proposed to help induce $\mathrm{C}-\mathrm{O}$ bond cleavage to give $\mathrm{CO}$ (REF. 42). c $\mid$ Azamacrocycles of $\mathrm{Ni}(9)^{50}$ and $\mathrm{Fe}(10)^{51}$, which feature proximal protic residues, perform $\mathrm{CO}_{2}$ reduction through cooperative catalysis. In both cases, the reduced metal serves as the nucleophile that attacks the $\mathrm{C}$ atom in $\mathrm{CO}_{2}$, with the protic moiety stabilizing the negatively charged $\mathrm{O}$ atoms that result. In the protein structures, $\mathrm{C}$ (grey), N (blue), O (red), P (gold), S (yellow), Fe (orange) and Ni (grey) atoms are shown in their respective colours. The protein scaffold is rendered in teal. Part a was drawn using data in REF. 48.

functionalities $-\mathrm{H}^{+}$relays that confer $\mathrm{CO}_{2}$ reduction activity on these complexes, although not at rates anywhere near those exhibited by the enzyme $e^{50,51}$. For both catalysts, it has been proposed that hydrogen-bond donors interact with $\mathrm{O}$ atoms in metal-bound $\mathrm{CO}_{2}$ to stabilize the otherwise labile adduct. The structures of mononuclear complexes 9 and 10 are so distinct from the active sites of [NiFe]-CODHase that they should not be considered biomimetics, although their mechanisms of action are somewhat similar to the native mechanism, such that further investigation is justifiable. Indeed, $\mathrm{CO}_{2}$ reduction is a challenging multielectron, multiproton conversion, one that can no doubt benefit from metal-ligand cooperative catalysis.

\section{Conclusions}

We now know in intimate detail the mechanisms of metal-ligand cooperative catalysis in a number of enzymes, notably those involved in the metabolism of $\mathrm{H}_{2}$, alcohols and perhaps $\mathrm{CO}_{2}$. The organic functional groups that participate in cooperative catalysis are typically found in the first or second coordination sphere with respect to the metal, often in locations more remote than expected. The examples of cooperative catalysis in nature provide inspiration for synthetic chemists to develop functional mimics that perform efficient catalysis by virtue of operating through pathways in which the metal and the ligand(s) interact directly with the substrate(s). Finding the best ways to capture the essential features of biocatalysis and translating them into useful technologies remains an exciting challenge awaiting further study.

Matthew D. Wodrich is at the Laboratory of Inorganic Synthesis and Catalysis, and the Laboratory for Computational Molecular Design, Institute of Chemical Sciences and Engineering, École Polytechnique Fédérale de Lausanne.

Xile Hu is at the Laboratory of Inorganic Synthesis and Catalysis, Institute of Chemical Sciences and Engineering, École Polytechnique Fédérale de Lausanne, Lausanne 1015, Switzerland. Correspondence to X.H. xile.hu@epfl.ch doi:10.1038/s4157-017-0099 Published online 13 Dec 2017

. Khusnutdinova, J. R. \& Milstein, D. Metal-ligand cooperation. Angew. Chem. Int. Ed. 54, 12236-12273 (2015)

2. van der Vlugt, J. I. Cooperative catalysis with first-row late transition metals. Eur. J. Inorg. Chem. 2012, 363-375 (2012).

3. Collman, J. P. Synthetic models for the oxygen-binding hemoproteins. Acc. Chem. Res. 10, 265-272 (1977).

4. Collman, J. P. \& Fu, L. Synthetic models for hemoglobin and myoglobin. Acc. Chem. Res. 32, 455-463 (1999).

5. Vignais, P. M., Billoud, B. \& Meyer, J. Classification and phylogeny of hydrogenases. FEMS Microbiol. Rev. 25, 455-501 (2001).

6. Lubitz, W., Ogata, H., Rüdiger, O. \& Reijerse, E. Hydrogenases. Chem. Rev. 114, 4081-4148 (2014).

7. Zampella, G., Greco, C., Fantucci, P. \& De Gioia, L. Proton reduction and dihydrogen oxidation on models of the $[2 \mathrm{Fe}]_{H}$ cluster of $[\mathrm{Fe}]$ hydrogenases. A density functional theory investigation. Inorg. Chem. 45 4109-4118 (2006).

8. Rauchfuss, T. B. Diiron azadithiolates as models for the [FeFe]-hydrogenase active site and paradigm for the role of the second coordination sphere. Acc. Chem. Res. 48, 2107-2116 (2015).

9. Schilter, D., Camara, J. M., Huynh, M. T., HammesSchiffer, S. \& Rauchfuss, T. B. Hydrogenase enzymes and their synthetic models: the role of metal hydrides. Chem. Rev. 116, 8693-8749 (2016).
10. Evans, R. M. et al. Mechanism of hydrogen activation by [NiFe] hydrogenases. Nat. Chem. Biol. 12, 46-50 (2016).

11. Berggren, G. et al. Biomimetic assembly and activation of [FeFe]-hydrogenases. Nature 499, 66-69 (2013).

12. Esselborn, J. et al. Spontaneous activation of [FeFe] hydrogenases by an inorganic [2Fe] active site mimic. Nat. Chem. Biol. 9, 607-609 (2013).

13. Schilter, D. \& Rauchfuss, T. B. And the winner is azadithiolate: an amine proton relay in the [FeFe] hydrogenases. Angew. Chem. Int. Ed. 52, 13518-13520 (2013).

14. Esselborn, J. et al. A structural view of synthetic cofactor integration into [FeFe]-hydrogenases. Chem Sci. 7, 959-968 (2016).

15. Siebel, J. F. et al. Hybrid [FeFe]-hydrogenases with modified active sites show remarkable residual enzymatic activity. Biochemistry 54, 1474-1483 (2015).

16. Shima, S. \& Thauer, R. K. A third type of hydrogenase catalyzing $\mathrm{H}_{2}$ activation. Chem. Rec. 7, 37-46 (2007)

17. Shima, S. \& Ermler, U. Structure and function of [Fe]hydrogenase and its iron-guanylylpyridinol (FeGP) cofactor. Eur. J. Inorg. Chem. 2011, 963-972 (2011)

18. Shima, S. et al. The crystal structure of [Fe]hydrogenase reveals the geometry of the active site. Science 321, 572-575 (2008).

19. Tamura, H. et al. Crystal structures of [Fe]hydrogenase in complex with inhibitory isocyanides: implications for the $\mathrm{H}_{2}$-activation site. Angew. Chem. Int. Ed. 52, 9656-9659 (2013).

20. Yang, X. \& Hall, M. B. Monoiron hydrogenase catalysis: hydrogen activation with the formation of a dihydrogen, $\mathrm{Fe}-\mathrm{H}^{\delta-} \ldots \mathrm{H}^{\delta+}-\mathrm{O}$, bond and methenyl$\mathrm{H}_{4} \mathrm{MPT}^{+}$triggered hydride transfer. J. Am. Chem. Soc. 131, 10901-10908 (2009).

21. Finkelmann, A. R., Stiebritz, M. T. \& Reiher, M. Kinetic modeling of hydrogen conversion at [Fe] hydrogenase active-site models. J. Phys. Chem. B 117, 4806-4817 (2013).

22. Finkelmann, A. R., Senn, H. M. \& Reiher, M. Hydrogen-activation mechanism of [Fe] hydrogenase revealed by multi-scale modeling. Chem. Sci. $\mathbf{5}$ 4474-4482 (2014).

23. Shima, S. et al. Reconstitution of [Fe]-hydrogenase using model complexes. Nat. Chem. 7, 995-1002 (2015).

24. Schultz, K. M., Chen, D. \& Hu, X. [Fe]-hydrogenase and models that contain iron-acyl ligation. Chem. Asian J. 8, 1068-1075 (2013).

25. Murray, K. A., Wodrich, M. D., Hu, X. \& Corminboeuf, C. Toward functional type III [Fe]hydrogenase biomimics for $\mathrm{H}_{2}$ activation: insights from computation. Chemistry 21, 3987-3996 (2015). 
26. $\mathrm{Xu}$, T. et al. A functional model of [Fe]-hydrogenase. J. Am. Chem. Soc. 138, 3270-3273 (2016).

27. Moore, C. M., Dahl, E. W. \& Szymczak, N. K. Beyond $\mathrm{H}_{2}$ : exploiting 2-hydroxypyridine as a design element from [Fe]-hydrogenase for energy-relevant catalysis. Curr. Opin. Chem. Biol. 25, 9-17 (2015).

28. Desguin, B. et al. A tethered niacin-derived pincer complex with a nickel-carbon bond in lactate racemase. Science 349, 66-69 (2015)

29. Xu, T., Bauer, G. \& Hu, X. A novel nickel pincer complex in the active site of lactate racemase. Chembiochem 17, 31-32 (2016).

30. Desguin, B., Soumillion, P., Hols, P., Hu, J. \& Hausinger, R. P. in The Biological Chemistry of Nickel (eds Zamble, D., Rowinska-Zyrek, M. \& Kozlowski, H.) 220-236 (Royal Society of Chemistry, 2017).

31. Cantwell, A. \& Dennis, D. Lactate racemase. Direct evidence for an $\alpha$-carbonyl intermediate. Biochemistry 13, 287-291 (1974)

32. Zhang, X. \& Chung, L. W. Alternative mechanistic strategy for enzyme catalysis in a $\mathrm{Ni}$-dependent lactate racemase (LarA): intermediate destabilization by the cofactor. Chemistry 23, 3623-3630 (2017)

33. Yu, M.-J. \& Chen, S.-L. From NAD ${ }^{+}$to nickel pincer complex: a significant cofactorevolution presented by lactate racemase. Chemistry 23, 7545-7557 (2017).

34. Eklund, H., Plapp, B. V., Samama, J.-P. \& Brädén, C.-I. Binding of substrate in a ternary complex of horse liver alcohol dehydrogenase. J. Biol. Chem. 257, 14349-14358 (1982)

35. Cook, P. F. \& Cleland, W. W. pH Variation of isotope effects in enzyme-catalyzed reactions. 2. Isotopedependent step not $\mathrm{pH}$ dependent. Kinetic mechanism of alcohol dehydrogenase. Biochemistry 20 , 1805-1816 (1981).

36. Eklund, H. et al. Three-dimensional structure of horse liver alcohol dehydrogenase at $2.4 \AA$ resolution J. Mol. Biol. 102, 27-59 (1976).

37. Ramaswamy, S., Eklund, H. \& Plapp, B. V. Structures of horse liver alcohol dehydrogenase complexed with $\mathrm{NAD}^{+}$and substituted benzyl alcohols. Biochemistry 33, 5230-5237 (1994).

38. Inoue, J., Tomioka, N., Itai, A. \& Harayama, S. Proton transfer in benzyl alcohol dehydrogenase during catalysis: alternate proton-relay routes. Biochemistry 37, 3305-3311 (1998)

39. Xu, T., Wodrich, M. D., Scopelliti, R., Corminboeuf, C. $\& \mathrm{Hu}, \mathrm{X}$. Nickel pincer model of the active site of lactate racemase involves ligand participation in hydride transfer. Proc. Natl Acad. Sci. USA 114, 1242-1245 (2017).
40. Dauth, A., Gellrich, U., Diskin-Posner, Y., Ben-David, Y. $\&$ Milstein, D. The ferraquinone-ferrahydroquinone couple: combining quinonic and metal-based reactivity. J. Am. Chem. Soc. 139, 2799-2807 (2017).

41. McSkimming, A., Chan, B., Bhadbhade, M. M. Ball, G. E. \& Colbran, S. B. Bio-inspired transition metal-organic hydride conjugates for catalysis of transfer hydrogenation: experiment and theory. Chemistry 21, 2821-2834 (2015).

42. Can, M., Armstrong, F. A. \& Ragsdale, S. W. Structure, function, and mechanism of the nickel metalloenzymes, $\mathrm{CO}$ dehydrogenase, and acetyl-CoA synthase. Chem. Rev. 114, 4149-4174 (2014).

43. Dobbek, H., Svetlitchnyi, V., Gremer, L., Huber, R. \& Meyer, O. Crystal structure of a carbon monoxide dehydrogenase reveals a [Ni-4Fe-5S] cluster. Science 293, 1281-1285 (2001).

44. Jeoung, J.-H. \& Dobbek, H. Carbon dioxide activation at the $\mathrm{Ni}$, Fe-cluster of anaerobic carbon monoxide dehydrogenase. Science 318, 1461-1464 (2007).

45. Lindahl, P. A. The Ni-containing carbon monoxide dehydrogenase family: light at the end of the tunnel? Biochemistry 41, 2097-2105 (2002).

46. Feng, J. \& Lindahl, P. A. Carbon monoxide dehydrogenase from Rhodospirillum rubrum: effect of redox potential on catalysis. Biochemistry 43 1552-1559 (2004)

47. Fesseler, J., Jeoung, J.-H. \& Dobbek, H. How the $\left[\mathrm{NiFe}_{4} \mathrm{~S}_{4}\right]$ cluster of $\mathrm{CO}$ dehydrogenase activates $\mathrm{CO}_{2}$ and $\mathrm{NCO}^{-}$. Angew. Chem. Int. Ed. 54, 8560-8564 (2015).

48. Kim, E. J., Feng, J., Bramlett, M. R. \& Lindahl, P. A Evidence for a proton transfer network and a required persulfide-bond-forming cysteine residue in Ni-containing carbon monoxide dehydrogenases. Biochemistry 43, 5728-5734 (2004).

49. Rao, P. V. \& Holm, R. H. Synthetic analogues of the active sites of iron-sulfur proteins. Chem. Rev. 104 527-560 (2004)

50. Beley, M., Collin, J.-P., Ruppert, R. \& Sauvage, J.-P. Electrocatalytic reduction of $\mathrm{CO}_{2}$ by Ni cyclam ${ }^{2+}$ in water: study of the factors affecting the efficiency and the selectivity of the process. J. Am. Chem. Soc. 108 7461-7467 (1986)

51. Costentin, C., Drouet, S., Robert, M. \& Savéant, J.-M. A local proton source enhances $\mathrm{CO}_{2}$ electroreduction to $\mathrm{CO}$ by a molecular Fe catalyst. Science $338,90-94$ (2012).

52. Rakowski DuBois, M. \& DuBois, D. L. The roles of the first and second coordination spheres in the design of molecular catalysts for $\mathrm{H}_{2}$ production and oxidation. Chem. Soc. Rev. 38, 62-72 (2009).

53. Wilson, A. D. et al. Nature of hydrogen interactions with $\mathrm{Ni}$ (II) complexes containing cyclic phosphine ligands with pendant nitrogen bases. Proc. Natl Acad. Sci. USA 104, 6951-6956 (2007).

54. Brazzolotto, D. et al. Nickel-centred proton reduction catalysis in a model of [NiFe] hydrogenase. Nat. Chem 8, 1054-1060 (2016).

55. Crabtree, R. H. Dihydrogen complexation. Chem. Rev. 116, 8750-8769 (2016).

56. DuBois, D. L. \& Bullock, R. M. Molecular electrocatalysts for the oxidation of hydrogen and the production of hydrogen - the role of pendant amines as proton relays. Eur. J. Inorg. Chem. 2011 1017-1027 (2011)

57. Xu, T., Chen, D. \& Hu, X. Hydrogen-activating models of hydrogenases. Coord. Chem. Rev. 303, 32-41 (2015)

58. Ginovska-Pangovska, B., Dutta, A., Reback, M. L., Linehan, J. C. \& Shaw, W. J. Beyond the active site: the impact of the outer coordination sphere on electrocatalysts for hydrogen production and oxidation. Acc. Chem. Res. 47, 2621-2630 (2014).

\section{Acknowledgements}

The authors thank the Swiss National Science Foundation for financial support (200020_172486/1). M.D.W. acknowledges C. Corminboeuf (École Polytechnique Fédérale de Lausanne, Switzerland) for financial support. G. Gryn'ova is acknowledged for artistic contributions.

\section{Author contributions}

Both authors contributed equally to the preparation of this manuscript.

\section{Competing interests statement}

The authors declare no competing interests.

\section{Publisher's note}

Springer Nature remains neutral with regard to jurisdictional claims in published maps and institutional affiliations.

How to cite this article

Wodrich, M. D. \& Hu X. Natural inspirations for metal-ligand cooperative catalysis. Nat. Rev. Chem. 2, 0099 (2018).

DATABASES

Protein Data Bank: http://www.rcsb.org/pdb/home/home.do 3DAG, 4UDX, 5UDB 